\title{
Integrated Tunable Magnetic RF Inductor
}

\author{
Marina Vroubel, Yan Zhuang, Behzad Rejaei, and Joachim N. Burghartz, Fellow, IEEE
}

\begin{abstract}
We demonstrate, for the first time to our knowledge, a passive, electrically tunable integrated radio frequency $(\mathbf{R F})$ inductor based on a planar solenoid with a thin-film ferromagnetic(FM) (NiFe) core. Variation of inductance is achieved by leading an additional dc current through the same device, thereby changing the effective permeability of the FM core. Tuning ranges (relative variations in inductance) of $85 \%, 35 \%$, and $20 \%$ are achieved at $0.1,1$, and $2 \mathrm{GHz}$, respectively, for inductances in the range of 1 to $150 \mathrm{nH}$.
\end{abstract}

Index Terms-Inductor, on-chip, radio frequency (RF), tunable circuits and devices.

\section{INTRODUCTION}

$\mathbf{E}$ XTENSIVE research is currently being conducted on the integration of inductors and capacitors used in impedance matching networks, $L C$-tanks, filters, and other applications in radio-frequency $(\mathrm{RF})$ silicon technology. Tunability of these $\mathrm{RF}$ passive components is highly desirable, as it gives the RF circuit designer the possibility to arrange for an adjustment to the optimum circuit operation at all termination conditions. That is e.g., helpful for maximizing the efficiency and linearity of a power amplifier by varying the input and output impedances. Electrically (pn-junction varactors; MOS-varactors) and micro-electromechanically (MEMS) tunable capacitors are already available in RF integration processes or are in development, while work on tunable inductors is leaping far behind. High quality factor and highly tunable active inductors have been reported [1], yet their widespread use in integrated $\mathrm{RF}$ circuits is impeded by high power consumption, complexity of power handling, high noise level, and nonlinear behavior. There are only a few MEMS approaches to tunable inductors so far [2]-[4]. Those components are of limited use for volume manufacturing due to the large area consumption, high mask count, and limited tuning range $(<18 \%[4])$.

In this letter we demonstrate, to our knowledge for the first time, a passive, electrically tunable integrated RF inductor.

\section{RESULTS AND DISCUSSION}

The operation of a planar RF solenoid with an FM core [5] is based on inducing a RF magnetic field perpendicular to the direction of the magnetization (easy axis) in the core [Fig. 1(a)]. The windings of the solenoid are built parallel to the easy axis,

Manuscript received September 14, 2004. This work was supported in part by the Foundation for Fundamental Research on Matter (FOM), The Netherlands. The review of this letter was arranged by Editor J. Sin.

The authors are with the Laboratory of High-Frequency Technology and Components, Delft Institute of Electronics and Submicron Technology (DIMES), Delft University of Technology, Delft, The Netherlands (e-mail: m.vroubel@ewi.tudelft.nl).

Digital Object Identifier 10.1109/LED.2004.839208

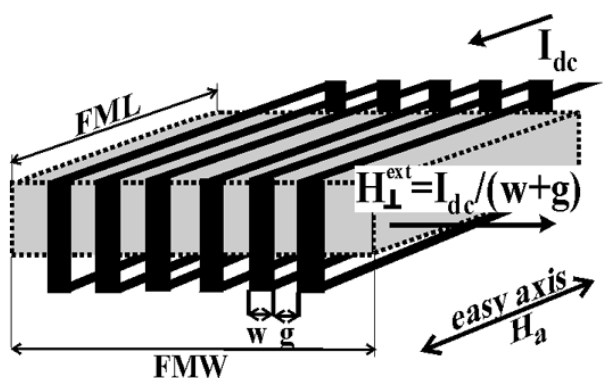

(a)

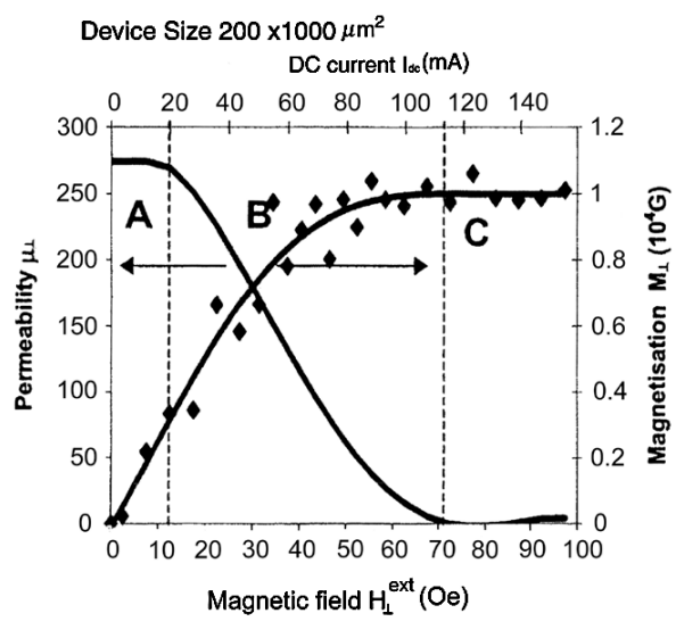

(b)

Fig. 1. (a) Sketch of a biased planar solenoid. $I_{\mathrm{dc}}$ denotes the dc-current through the coil inducing a static magnetic field $H_{\perp}^{\text {ext }}$. The magnetization is mainly orientated along the easy axis of the FM film; $\bar{H}_{a}$ denotes the anisotropy field. FMW and FML denote the width and length of the ferromagnetic core, correspondingly. (b) Measured B-H curves (rhomb-markers), the least square fits for the measured results (solid lines), and permeability extracted from the least square fits (solid lines). The secondary (upper) horizontal axis gives the values of the dc bias current $I_{\mathrm{dc}}$, with the associated bias magnetic field given on the bottom horizontal axis $H_{\perp}^{\text {ext }}=I_{\mathrm{dc}} /(w+g)$, where $w=10 \mu \mathrm{m}$ and $g=10 \mu \mathrm{m}$, respectively, as given in the paper. The letters A, and C correspond to the regions of (constant) high and low permeability, respectively. In region B, the permeability changes due to nonuniform distribution of the magnetization in the core.

which lies in the plane of the film for thin layers. In this configuration, the permeability $\mu_{\perp}$ of the ferromagnetic (FM) film depends on the internal magnetocrystalline anisotropy and demagnetization fields, as well as on an external dc field $H_{\perp}^{\text {ext }}$ applied perpendicular to the easy axis, i.e., along the hard axis. At frequencies sufficiently lower than the ferromagnetic resonance frequency [6], $\mu_{\perp}$ can be obtained from the magnetization curve according to $\mu_{\perp}=1+\partial M_{\perp} / \partial H_{\perp}^{\text {ext }}$, where $M_{\perp}$ and $H_{\perp}^{\text {ext }}$, respectively, denote the magnetization and the external dc field in the hard-direction.

Fig. 1(b) shows a typical, experimental magnetization curve and the extracted permeability for a thin FM sample. One can 
clearly distinguish three regions denoted by A, B, and C. In regions $\mathrm{A}$ and $\mathrm{C}$, the permeability practically remains constant with increasing magnetic field, with a high permeability in A and a low permeability in C. In the intermediate region (B), the permeability decreases with increasing magnetic field. The strong dependence of the permeability on the external magnetic field in region B can be explained by taking into account the nonuniform distribution of magnetization inside the core [7]. Furthermore, the threshold field required for the onset of this behavior depends on the mutual balance of internal shape-induced demagnetizing fields in the easy and hard directions and for the set of rectangular FM samples of either the fixed width (FMW) or the fixed length (FML) is minimum for square samples $(F M L=F M W)$ [7], [8]. The strong dependence of the permeability on the external dc field in the intermediate region (B) lies at the basis of the tunable inductor. A dc current $I_{\mathrm{dc}}$ flowing through the windings of the planar solenoid induces a dc magnetic field along the hard axis approximately given by $H_{\perp}^{\text {ext }}=I_{\mathrm{dc}} /(w+g)$, where $w$ is the width of the metal wires and $g$ is the spacing between wires [Fig. 1(a)]. Thus, applying a dc bias current in addition to the RF signal changes the FM permeability and the inductance of the device. In order to verify this principle and to study the effect of the shape of the FM core, we have fabricated various planar solenoids with a $0.5-\mu \mathrm{m}$-thick $\mathrm{NiFe}$ core on a silicon substrate. Vias were used to connect parallel metal strips on two metal layers 3 - $\mu$ m-thick $\mathrm{Al}$, each separated from the $\mathrm{NiFe}$ core by a $1-\mu \mathrm{m}$-thick $\mathrm{SiO}_{2}$ layer. The track width and spacing of the metal wires were $w=10 \mu \mathrm{m}$ and $g=10 \mu \mathrm{m}$, respectively, for all coils, yielding the same dc field in all cases for a given $I_{\mathrm{dc}}$.

Fig. 2 shows the measured inductance $L$ and quality factor $Q$ as functions of frequency and dc bias current for three solenoids with FM cores of the same width (FMW) of $200 \mu \mathrm{m}$ but different lengths (FML). A relative variation in inductance $\Delta L / L$ ranging up to $50 \%$ at $0.1 \mathrm{GHz}$ and up to $20 \%$ at $2 \mathrm{GHz}$ [Fig. 2(b)], was observed by applying bias currents up to $100 \mathrm{~mA}$. Furthermore, $\Delta L / L$ increased, achieving the highest value for this set of inductors, by bringing the aspect ratio (FMW/FML) to one [Fig. 2(b)], in agreement with the expected behavior of the demagnetization field inside the magnetic core [7], [8]. The observed gradual drop in $L$ with increasing frequency [Fig. 2(b) and (c)] is mainly due to eddy currents in the metallic NiFe core [5]. Note, that biasing the coils yields higher $Q$ 's at high frequencies [Fig. 2(b) and (c)] due to the decrease in effective permeability, leading, in turn, to lower eddy current loss. Among the fabricated devices, the largest tuning range $(85 \%$ reduction of inductance at $0.1 \mathrm{GHz}$ ) was observed for a coil built using a wide (FMW $=1000 \mu \mathrm{m}) \mathrm{FM}$ core [Fig. 3(a)], which results in a low demagnetizing field in the direction of the hard axis. Nevertheless, in this case the large size of the coil limits the resonance frequency and, thus, the operating frequency range [5].

The inductors presented in this paper have limited quality factors $(Q<2)$ and high level of dc power consumption $\left(0.015-0.3 \mathrm{~W}\right.$ for $\left.I_{\mathrm{dc}}=100 \mathrm{~mA}\right)$. These shortcomings can be mainly attributed to the high conductivity of the FM materials

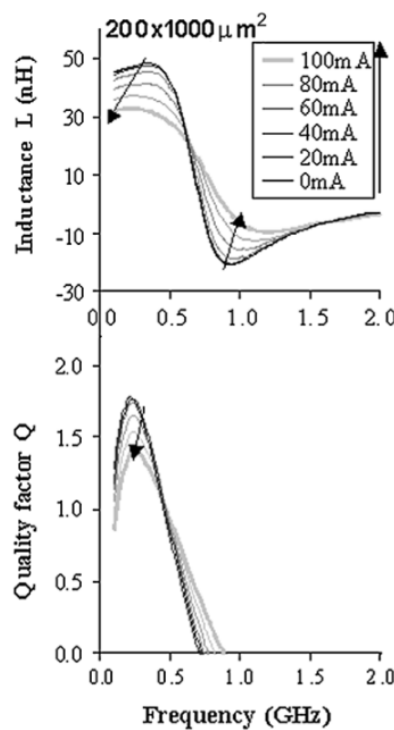

(a)

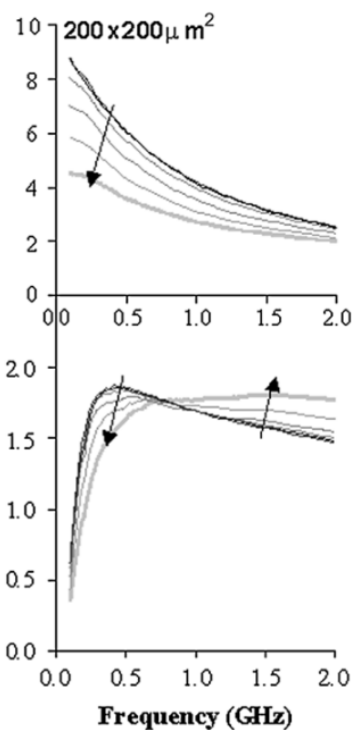

(b)

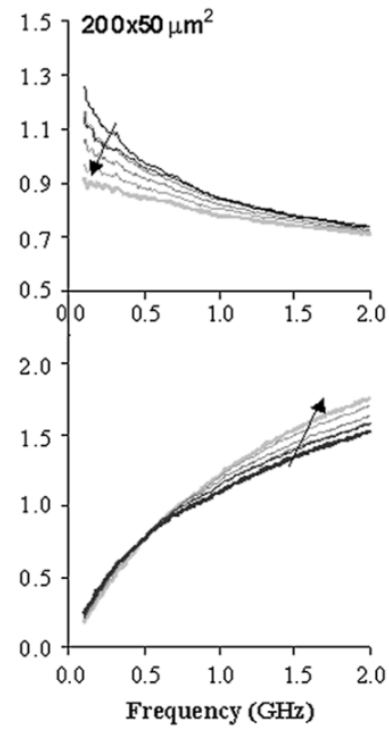

(c)

Fig. 2. Inductance and quality factors versus frequency for different values of $I_{\mathrm{dc}}$ for solenoids with different FM-core lengths (FML) but the same core-width $(\mathrm{FMW}=200 \mu \mathrm{m})$

and nonefficient design of the metal coils. Our simulations have shown, that the use of novel high-resistivity FM materials with conductivity lower than $10^{5} \mathrm{~S} / \mathrm{m}$ [9] leads to quality factors higher than 15 for permeabilities above 50 [Fig. 3(b)].

To ensure a broad tuning range, one, in principle, has to reduce the anisotropy field $H_{a}$. However, this will decrease the ferromagnetic resonance frequency, above which the ferromagnetic inductor does not function efficiently. Our simulations have shown, that a field of $H_{a}=60 \mathrm{Oe}$ is a good compromise to obtain a tuning range of $87 \%$ with a dc power consumption of $0.7 \mathrm{~mW}$ at $1 \mathrm{GHz}$ for the FM core with conductivity of $10^{5}$ $\mathrm{S} / \mathrm{m}$ [Fig. 3(c)]. Relying on the high-frequency permeability measurement, we expect only a slight deterioration of tuning range $(>80 \%)$ at $2 \mathrm{GHz}$ for a low conductive magnetic core with magnetic characteristics like mentioned above. 


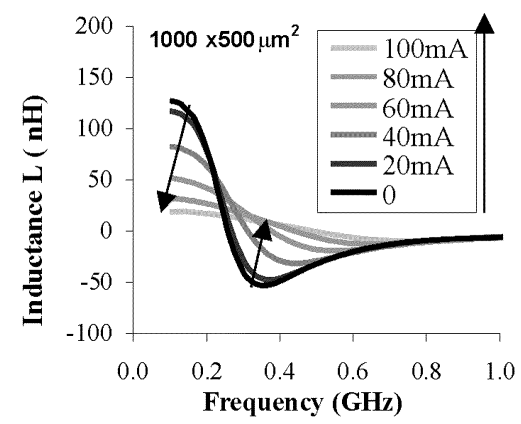

(a)

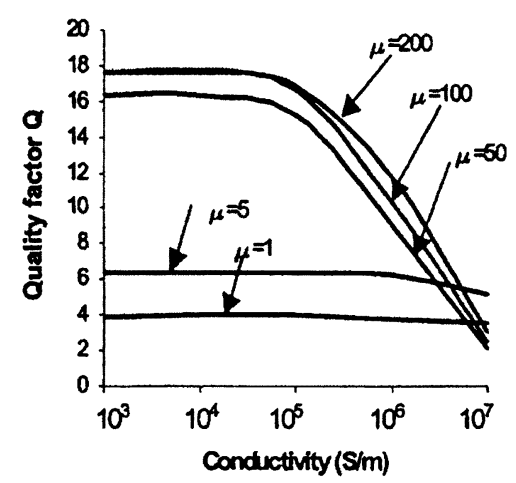

(b)

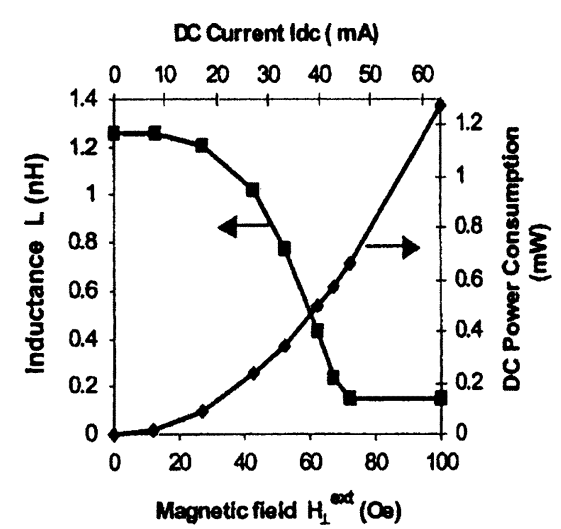

(c)

Fig. 3. (a) Inductance versus frequency for different values of $I_{\mathrm{dc}}$ for an inductor with a large $\left(1000 \times 500 \mu \mathrm{m}^{2}\right)$ magnetic core, characterized by small demagnetizing fields. The tuning range equals $85 \%$ for $I_{\mathrm{dc}}=100 \mathrm{~mA}$ at $0.1 \mathrm{GHz}$. (b) Simulated $Q$ of five-turn 50 by $50 \mu \mathrm{m}$ solenoid as a function of FM film conductivity and permeability at $2 \mathrm{GHz}$. The FM layer is $1 \mu \mathrm{m}$ thick, separated from the coil by a $0.5-\mu \mathrm{m}$-thick insulation layer. The coil is built from 5- $\mu \mathrm{m}$-thick copper with $w=5 \mu \mathrm{m}$ and $\mathrm{g}=3 \mu \mathrm{m}$. (c) Simulated inductance and dc power consumption as functions of dc current and external magnetic field at $1 \mathrm{GHz}$ for the same solenoid as in Fig. 3(b) with conductivity of FM core equals to $10^{5} \mathrm{~S} / \mathrm{m}$ and permeability taken from Fig. 1(b).

\section{CONCLUSION}

In conclusion, we have demonstrated to our knowledge the first passive, electrically tunable inductors with a very high tuning range of up to $85 \%$ at low frequency and up to $35 \%$ at $1 \mathrm{GHz}$. Substantial further improvements in dc bias current level, physical size, and quality factor can be expected from optimized coil and FM film structural designs. The quality factors can be potentially increased up to $\sim 17$ by using low-conductivity $\left(<10^{5} \mathrm{~S} / \mathrm{m}\right) \mathrm{FM}$ cores. The dc power consumption can be decreased to $0.7 \mathrm{~mW}$ for a $50 \times 50 \mu \mathrm{m}^{2}$ inductor by optimizing the coil geometry, while securing the high $(>80 \%)$ tunability range.

\section{ACKNOWLEDGMENT}

The authors would like to thank A. M. Crawford of Stanford University for performing B-H measurements.

\section{REFERENCES}

[1] R. Mukhopadhyay, Y. Park, P. Sen, N. Srirattana, J. S. Lee, S. Nuttinck, A. J. Joseph, J. D. Cressler, and J. Laskar, "Reconfigurable RFICs for frequency-agile VCOs in Si-based technology for multi-standard applications," in Proc. MTT-S , vol. 3, 2004, pp. 1489-1492.

[2] I. Zine-El-Abidene, M. Okoniewski, and J. G. McRory, "A new class of tunable RF MEMS inductors," in Proc. ICMENS, 2003, pp. 1-3.

[3] X. Q. Sun, S. Zhou, and W. N. Carr, "A micro variable inductor chip using MEMS relay," in Proc. Int. Conf. Solid-State Sensors Actuators, 1997, pp. 1137-1140.

[4] V. M. Lubecke, B. Barber, E. Chan, D. Lopez, M. I. Gross, and P. Gammel, "Self-assembling MEMS variable and fixed RF inductors," IEEE Trans. Microwave Theory Techn., vol. 49, pp. 2093-2097, Nov. 2001.

[5] Y. Zhuang, M. Vroubel, B. Rejaei, E. Boellaard, and J. N. Burghartz, "Ferromagnetic RF inductors and transformers for standard CMOS/BiSMOS," in IEDM Tech. Dig., 2002, pp. 18.7.1-18.7.4.

[6] A. G. Gurevich and G. A. Melkov, Magnetization Oscillations and Waves. Boca Raton, FL: CRC, 1996, vol. 24-25, pp. 14-17.

[7] M. Vroubel, Y. Zhuang, B. Rejaei, J. N. Burghartz, A. M. Crawford, and S. X. Wang, "Calculation of shape anisotropy for micropatterned thin Fe-Ni films for on-chip RF applications," IEEE Trans. Magn., vol. 40, pp. 2835-2837, July 2004.

[8] E. C. Stoner and E. P. Wohlfarth, "A mechanism of magnetic hysteresis in heterogeneous alloys," IEEE Trans. Magn., vol. 27, pp. 3475-3517, Apr. 1991.

[9] M. Munakata, M. Namikawa, M. Motoyama, M. Yagi, Y. Shimagda, M. Yamaguchi, and K.-I. Arai, "Magnetic properties and frequency characteristics of $(\mathrm{Co} \mathrm{FeB})_{x}-(\mathrm{SiO} 1.9)_{1-x}$ and $\mathrm{CoFeB}$ films for $\mathrm{RF}$ application," Proc. Trans. Magn. Soc., vol. 2, pp. 388-393, Dec. 2002. 